\title{
A special issue on glucose transporters in health and disease
}

\author{
Hermann Koepsell ${ }^{1} \cdot$ Volker Vallon $^{2,3,4}$ (D) \\ Received: 16 July 2020 / Revised: 16 July 2020 / Accepted: 31 July 2020 / Published online: 11 August 2020 \\ (C) Springer-Verlag GmbH Germany, part of Springer Nature 2020
}

Glucose is the most important energy carrier and building block in all kingdoms of life. As a consequence, the normal function of plants, bacteria, and animal organs depends on the continuous generation and/or supply and uptake of glucose. The goal of this Pflüger's Special Issue is to summarize key concepts related to glucose transport in human health and disease, covering established paradigms but also recent developments and new insights that make this Special issue a timely endeavor. To put the topic in a larger perspective, we start the series with the role of sugar transport in plants and bacteria (Fig. 1).

In contrast to animals and most bacteria, plants can synthesize a surplus of sugars. As a consequence, plants are autotrophic organisms and basically provide all food on earth, either directly or indirectly. The contribution by Geiger [4] discusses the role of glucose as the main building block for cellulose synthesis but also as a signaling molecule to adjust plant growth and survival to metabolic status. The author introduces the proton-coupled sugar transport proteins as the plant counterparts of the animal sodium-dependent glucose transporters (SGLTs), and discusses how they facilitate the fine-tuned transport across membranes of the plant body.

Most animals can resorb only a small number of monosaccharides from the intestine, mostly D-glucose, D-galactose,

This article is part of the Special Issue on "Glucose Transporters in Health and Disease"

Hermann Koepsell

hermann@koepsell.de

Volker Vallon

vvallon@ucsd.edu

1 Institute for Anatomy and Cell Biology, University of Würzburg, Würzburg, Germany

2 Division of Nephrology and Hypertension, Department of Medicine, University of California San Diego, La Jolla, CA, USA

3 Department of Pharmacology, University of California San Diego, La Jolla, CA, USA

4 VA San Diego Healthcare System, San Diego, CA, USA and D-fructose. Other sugars and dietary fibers are mainly degraded and metabolized by the bacteria in the lower intestine. The contribution by Jeckelmann and Ernie [8] describes the structures and mechanisms of bacterial sugar transporters, and how they can import structurally different sugars. The authors also discuss how some bacterial sugar transporters are hijacked for import of bacteriophage DNA and antibacterial toxins, and how the presence and uptake of sugars may affect pathogenicity.

The contribution by Holman [7] introduces the first major family of mammalian glucose transporters, namely the SLC2 genes, which encode for facilitative glucose transporters of the GLUT protein family. The preferred substrates of these transporters are usually a hexose, but some GLUTs transport urate, dehydro-ascorbate, or myo-inositol. The main physiological functions of each of these GLUT proteins are briefly outlined. New insights from crystal structures revealed how the unique protein fold of these proteins enables the catalysis of transport. The author discusses how the availability of GLUT crystal structures has revealed drug targetable properties, which facilitates the search for specific inhibitors and substrates.

The molecular biology of the second major family of mammalian glucose transporters, namely the SLC5 genes, which encode for sodium-dependent glucose transporters of the SGLT family, is reviewed by Gyimesi, Hediger, and colleagues [6]. The 12 different human members of this family mediate the transport not only of sugars but also of vitamins, amino acids, or smaller organic ions such as choline. The review discusses the structural biology and molecular architecture of SGLT1 and SGLT2. The development of therapeutic inhibitors for SGLT1 and SGLT2 is described, including the pathophysiological basis and molecular modeling of drugtarget interactions.

The function and regulation of dietary sugar uptake in the intestine is discussed by Koepsell [9]. This includes the role of SGLT1 and GLUT2 for the absorption of D-glucose and Dgalactose and the secretion of enterohormones, while GLUT5 is relevant for D-fructose absorption. The author also describes the pathophysiological role of these transporters in diseases like glucose-galactose malabsorption, fructose intolerance, 


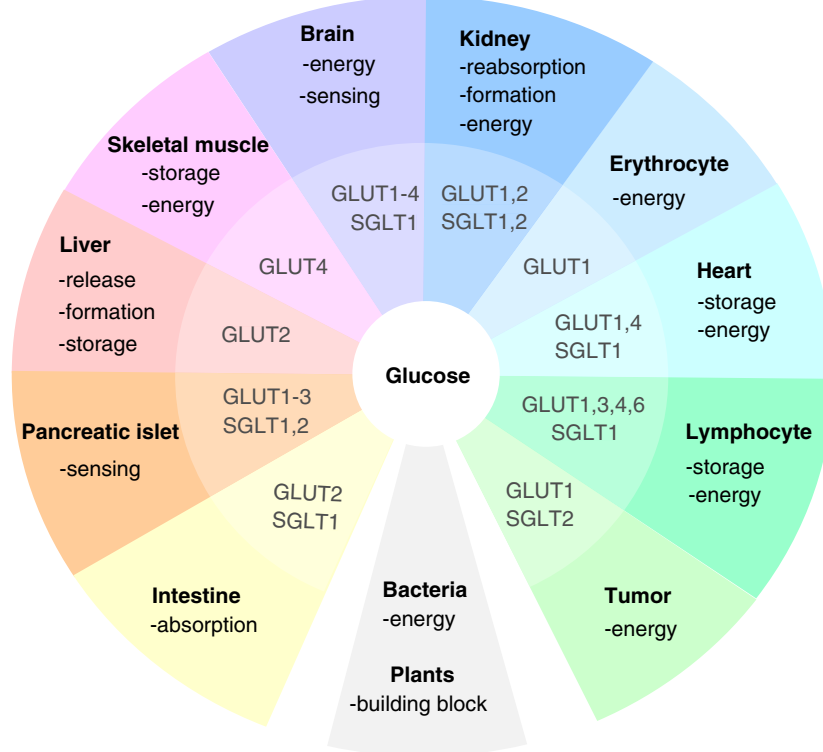

Fig. 1 Role of glucose transporters. Depicted are the main human glucose transporters and the function of glucose in a given organ or cell

diabetes, and small intestinal inflammation, as well as their therapeutic implications for parental nutrition, bariatric surgery, metformin treatment, or as a direct or indirect drug target.

Pancreatic islets sense extracellular glucose, e.g., the increase following food intake, and play a critical role in glucose-dependent insulin and glucagon release to maintain blood glucose homeostasis. The review by Berger and Zdzieblo [1] discusses the proven and putative role of glucose transporters in pancreatic islet physiology. This includes GLUT2 as the main glucose transporter in insulin-secreting $\beta$-cells in rodents, while recent data propose a role of GLUT1 and GLUT3 in humans. The authors also outline the latest information on the expression of SGLT1 and SGLT2 in islet $\alpha$-cells and their potential role in the regulation of glucagon release, and review the role of islet glucose transporters in the pathophysiology of diabetes.

Homeostatic control of blood glucose levels involves hormone-regulated glucose uptake in the liver, skeletal muscle, and adipose tissue, and release from the liver. The review by Chadt and Al-Hasani [3] outlines the importance of GLUT2 as the main regulator of hepatic hexose flux, and of GLUT4 as the workhorse in insulin- and contractionstimulated glucose uptake in skeletal muscle. The authors describe the molecular mechanisms how insulin controls glucose transport and its relation to insulin resistance and impaired glycemic control in type 2 diabetes. They discuss the role of circulating metabolites, hormone-like molecules, and potential other glucose transporters, and point out significant knowledge gaps.

Glucose transporters carry D-glucose across the bloodbrain barrier and deliver it to astrocytes and neurons. The review by Koepsell [10] outlines the expression of GLUT1, GLUT2, GLUT3, GLUT4, and SGLT1 in the human brain. Their individual and specific roles in energy supply to neurons, glucose sensing, central regulation of systemic glucose homeostasis and feeding, as well as learning and memory formation are discussed. The pathophysiological relevance is illustrated by loss-of-function mutations in the GLUT1 deficiency syndrome, and conditions that alter the expression of glucose transporters in the brain like diabetes mellitus, Alzheimer's disease, stroke, and traumatic brain injury.

The kidneys filter large amounts of glucose, and the tubular system reabsorbs all filtered glucose to prevent the loss of this valuable fuel. The review by Vallon [12] describes how SGLT2, SGLT1, and GLUT2 retain filtered glucose in proximal tubules, while uptake of D-glucose from the blood side via GLUT1 energizes transport work in distal tubular segments. The regulation of glucose reabsorption and coordination with glucose formation are discussed, as well as a new role of SGLT1 in acute kidney injury and for glucose sensing at the macula densa. The article explains the maladaptive role of renal glucose retention in hyperglycemia and the unexpected logic of SGLT2 inhibitors as new antihyperglycemic and nephroprotective drugs.

The key role of glucose for the functionality and integrity of red blood cells is outlined by Guizouarn and Allegrini [5]. Glucose transport in vertebrate erythrocytes is mediated by GLUT1 (in humans) or GLUT4, which also take up dehydroascorbic acid. The authors discuss the molecular regulation of glucose transport in erythrocytes as well as the deleterious consequences for their morphology, deformability, and lifespan when glucose metabolism is impaired, including the specific pathophysiologies of GLUT1 mutations, diabetes, and malaria.

As discussed by Bertrand and colleagues [2], glucose is also one of the main energy substrates of the heart, and its uptake and use is fine-tuned by the complex regulation of GLUT4 and GLUT1. SGLT1-mediated D-glucose uptake may not be linked to energy production but the formation of reactive oxygen species. The authors outline the distinct pathophysiological roles of glucose transporters in cardiac diseases like cardiac hypertrophy, diabetic cardiomyopathy, and heart failure, as well as proposed therapies targeting glucose transporters.

Energy supply in activated and proliferating lymphocytes largely depends on glucose. Lang and colleagues [11] discuss the role of GLUT1, GLUT3, GLUT4, GLUT6, and in particular SGLT1 in glucose transport in lymphocytes. Since SGLT1 accumulates glucose against glucose gradients, it is also effective at low extracellular glucose concentrations, which may be relevant in tumors and infected or inflamed tissues. Gene knockout of sglt1 in mice reduced bacterial clearance and increased host mortality. The authors point out the need for a better understanding of glucose transport in 
lymphocytes in the host defense against tumors and bacterial infections, including a potential role of SGLT1 in glucose uptake and glycogen storage.

Finally, also tumors have a high rate of glucose uptake to fuel the glycolysis that plays a central role in tumor metabolism and growth. This is discussed by Wright [13] including a role for GLUT1 and the successful clinical use of its substrate 2FDG in Positron-Emission-Tomography (PET) imaging to stage specific tumors. The author's recent work introduced a new radiotracer and SGLT substrate, Me4FDG, for PET studies to image the role of SGLTs in tumor glycolysis. Moreover, he describes that SGLT2 is expressed in pancreatic and prostate tumors and glioblastomas with potential therapeutic implications for SGLT2 inhibitors.

We hope that the presented individual reviews of this Special issue and their combined content will be well received. We thank the authors who contributed their time and commitment to this Special Issue and thereby laid the basis for its success.

Acknowledgments The authors thank Eva Vallon for expert graphical support.

Funding information VV was supported by NIH grants R01DK112042, R01DK106102, R01HL142814, RF1AG061296, the UAB/UCSD O'Brien Center of Acute Kidney Injury NIH-P30DK079337, and the Department of Veterans Affairs.

\section{Compliance with ethical standards}

Competing interests Over the past 36 months, VV has served as a consultant and received honoraria from Astra-Zeneca, Boehringer Ingelheim, and Retrophin, and received grant support for investigatorinitiated research from Astra-Zeneca, Bayer, Boehringer Ingelheim, Janssen Pharmaceutical, Kyowa-Kirin, and Novo-Nordisk.

\section{References}

1. Berger C, Zdzieblo D (2020) Glucose transporters in pancreatic islets. Pflugers Arch - Eur J Physiol. https://doi.org/10.1007/ s00424-020-02383-4

2. Bertrand L, Auquier J, Renguet E, Ange M, Cumps J, Horman S, Beauloye C (2020) Glucose transporters in cardiovascular system in health and disease. Pflugers Arch - Eur J Physiol

3. Chadt A, Al-Hasani H (2020) Glucose transporters in adipose tissue, liver, and skeletal muscle in metabolic health and disease. Pflugers Arch - Eur J Physiol. https://doi.org/10.1007/s00424020-02417-x

4. Geiger D (2020) Plant glucose transporter structure and function. Pflugers Arch - Eur J Physiol

5. Guizouarn H, Allegrini B (2020) Erythroid glucose transport in health and disease. Pflugers Arch - Eur J Physiol. https://doi.org/ 10.1007/s00424-020-02406-0

6. Gyimesi G, Gimenez JP, Kanai Y, Hediger MA (2020) Sodiumcoupled glucose transport, the SLC5 family and therapeutically relevant inhibitors: from molecular discovery to clinical application. Pflugers Arch - Eur J Physiol

7. Holman GD (2020) Structure, function and regulation of mammalian glucose transporters of the SLC2 family. Pflugers Arch - Eur J Physiol. https://doi.org/10.1007/s00424-020-02411-3

8. Jeckelmann JM, Erni B (2020) Transporters of glucose and other carbohydrates in bacteria. Pflugers Arch - Eur J Physiol. https://doi. org/10.1007/s00424-020-02379-0

9. Koepsell H (2020) Glucose transporters in small intestine in health and disease. Pflugers Arch - Eur J Physiol. https://doi.org/10.1007/ s00424-020-02439-5

10. Koepsell H (2020) Glucose transporters in brain in health and disease. Pflugers Arch - Eur J Physiol. https://doi.org/10.1007/s00424020-02441-x

11. Lang F, Singh Y, Salker MS, Ma K, Pandyra AA, Lang PA, Lang KS (2020) Glucose transport in lymphocytes. Pflugers Arch - Eur J Physiol. https://doi.org/10.1007/s00424-020-02416-y

12. Vallon V (2020) Glucose transporters in the kidney in health and disease. Pflugers Arch - Eur J Physiol. https://doi.org/10.1007/ s00424-020-02361-w

13. Wright EM (2020) SGLT2 and cancer. Pflugers Arch - Eur J Physiol

Publisher's note Springer Nature remains neutral with regard to jurisdictional claims in published maps and institutional affiliations. 